\title{
Motor-learning Impairment by Amantadine in Healthy Volunteers
}

\author{
Abdallah Hadj Tahar, ${ }^{1,2}$, Pierre J Blanchet ${ }^{3}$ and Julien Doyon*,1,2 \\ 'Department of Psychology, University of Montreal, Downtown, Montreal, Que., Canada; ${ }^{2}$ Institut Universitaire de Gériatrie de Montréal, \\ Montreal, Que., Canada; ${ }^{3}$ Department of Stomatology, Faculty of Dental Medicine, University of Montreal \& Andre-Barbeau Movement \\ Disorders Unit, Montreal, Que., Canada
}

\begin{abstract}
NMDA receptor antagonists impair learning and memory in animal models, presumably by inhibiting long-term potentiation in the motor cortex. Human studies are limited and restricted by the paucity of safe NMDA antagonists. Here, we investigated the contribution of glutamatergic neurotransmission to the capacity of acquiring motor-adaptation learning in humans. In a double-blind design, $200 \mathrm{mg}$ of amantadine (a low-affinity NMDA receptor channel blocker) or a matching placebo were given orally to groups of 14 and I3 human healthy young volunteers, respectively. Blood samples were collected $3 \mathrm{~h}$ after treatment to assay plasma concentrations, and the subjects were then tested using a motor-adaptation paradigm consisting of an eight-target-pointing task. To rule out drug-related generalized impairments such sedation, tests measuring motor dexterity and attention were also administered pre- and post-treatment. Comparison of the mean performance levels on the motor-adaptation task revealed that subjects in the amantadine group performed at a lower level than those in the placebo group, but this difference did not reach significance. Interestingly, however, despite plasma amantadine concentrations being relatively low, ranging from 2.09 to $4.74 \mu \mathrm{M}$ (mean $=3.3 \mu \mathrm{M}$ ), they nevertheless correlated negatively with motor learning. Furthermore, when the amantadine group was divided into low-performance and high-performance subgroups, subjects in the former subgroup displayed mean amantadine concentrations 36\% higher than the latter subgroup, and performed significantly worser than the placebo group. No change in performance was found on the motor-dexterity and attention tests. Altogether, our results lend support to the hypothesis that normal NMDA receptor function is necessary for the acquisition of motor adaptation.

Neuropsychopharmacology (2004) 29, 187-194, advance online publication, 8 October 2003; doi: 10.1038/sj.npp. 1300317
\end{abstract}

Keywords: motor learning; adaptation; amantadine; NMDA receptor; glutamate; human

\section{INTRODUCTION}

In everyday life, humans use a variety of motor skills, which refer to the process by which movements, either produced alone or in sequence, come to be performed effortlessly through extensive periods of practice (Willingham, 1998). In experimental conditions, these skills often fall into two categories: motor sequence learning and motor adaptation (Doyon et al, 2003). While the first measures the incremental acquisition of movements into a well-executed behavior, the second tests our capacity to compensate for environmental changes (Shadmehr and Holcomb, 1997; Doyon et al, 2003). Without this type of learning, we would not be able to play a violin, develop controlled artistic movements or drive a car. Several studies, particularly those using brain-imaging techniques, have highlighted the

\footnotetext{
*Correspondence: Dr J Doyon, Department of Psychology, University of Montreal, PO Box 6128, Downtown, Montreal, Que., Canada H3C 3J7, Tel: + 5 I4 343 6502; Fax: + 5 I 4343 5787, E-mails: julien.doyon@umontreal.ca or abdallah.hadj.tahar@umontreal.ca Received 01 April 2003; revised 06 August 2003; accepted 12 August 2003

Online publication: 26 August 2003 at http://www.acnp.org/citations/ Npp06250303028/default.pdf
}

neuroanatomical substrate and, to a certain extent, the neural networks responsible for the acquisition of a new motor skill (for a review, see Doyon and Ungerleider, 2002). However, the physiological mechanisms of this type of procedural learning remain elusive. Learning-related improvements in motor tasks are thought to be related to the plasticity of the motor system (Jackson and Lemon, 2001; Li et al, 2001), and may also share common mechanisms with those of functional recovery after stroke (Butefisch et al, 1995; Nudo et al, 1996).

Using a pharmacological approach, some investigators have recently begun to elucidate the neurobiological bases of motor learning (Butefisch et al, 2000; Donchin et al, 2002), and have proposed that this type of memory may be mediated by synaptic plasticity such as long-term potentiation (LTP). In fact, LTP is considered by many to be one of the main physiological mechanisms subserving the memory process (Malenka and Nicoll, 1999). Induction of LTP requires the activation of glutamate $N$-methyl-D-aspartate (NMDA) receptors, notably in the hippocampus (Bliss and Collingridge, 1993), but also in several other regions of the brain including the motor cortex (Hess et al, 1996; Kitagawa et al, 1997) and the striatum (Charpier and Deniau, 1997; Centonze et al, 1999, 2001; Spencer and Murphy, 2000). 
Thus, glutamate via NMDA receptors may play a major role in motor learning.

In the present experiment, we attempted to evaluate the impact of NMDA receptor blockade on a new motor-skilllearning paradigm (ie motor adaptation) by using amantadine in healthy human volunteers. Amantadine is a drug administered to patients with Parkinson's disease (Verhagen Metman et al, 1998; Blanchet et al, 2003), blocking the NMDA receptor ion channel (Kornhuber et al, 1991). A version of the eight-target-pointing task was used for the evaluation of this form of motor learning (ie Flament $e t$ al, 1996; Shadmehr and Holcomb, 1997). We hypothesized that this pharmacological manipulation would interfere with the motor-learning process.

\section{MATERIAL AND METHODS}

\section{Subjects and Methodological Considerations}

The study protocol was approved by the Ethics Committee of the University of Montreal Hospital Centre (CHU Montreal). Healthy young volunteers were divided into two groups (13 subjects under placebo, 14 subjects under amantadine), and were matched for age, sex, and level of education (Table 1). They were tested after they provided written informed consent. All subjects were right-handed, as tested with the Edinburgh Handedness Inventory (Oldfield, 1971). In an effort to standardize the motor skill in using a joystick, experts in video games were excluded. Participants with experience in playing musical instruments were included because such abilities involve motor-sequence learning rather than motor adaptation. Other habits, such as smoking, coffee intake, drugs (birth control pills), or any psychostimulant agents, were not permitted before and during testing. The subjects were allowed to eat a light lunch during the day of the testing. Timing of the testing during the day was comparable in both groups. The participants usually took $200 \mathrm{mg}$ of amantadine or placebo between 0900 and $1300 \mathrm{~h}$ and the motor-adaptation task, administered over a period of $40 \mathrm{~min}$, was performed $3 \mathrm{~h}$ later, that is, between 1200 and $1600 \mathrm{~h}$.

\section{Procedure: Experimental Tasks}

A motor-adaptation paradigm consisting of an eight-targetpointing task, created on a platform Power-builder 6.0 licensed by Microsoft, was used (Figure 1). In this setup, the subject used a lever-operated pointer to follow an elliptical (nonlinear) path between points appearing on a computer screen. The starting point was indicated by a white circle $0.75 \mathrm{~cm}$ in diameter, which appeared in the center of the screen. A cross-hair cursor was superposed over the starting point. A line $(0.5 \mathrm{~cm}$ thick $)$ elliptical in shape (radius $2.5 \mathrm{~cm}$ ) indicated the direction of the path to follow. Eight red spots were arranged, equidistant, on a circle with a radius of $10 \mathrm{~cm}$. The subject's task was to attain the targets following a convex-shaped path the most accurately and rapidly possible, and within a time limit of $3 \mathrm{~s}$. The targets could be reached in two ways: (1) a direct mode (DM), in which the direction of movement of the lever and that of the computer cursor were matched. This condition was used as a familiarization condition, and consisted of a brief period of practice allowing for subject selection (inclusion criteria); (2) an indirect mode (IM), in which the directions of the joystick and cursor were opposed, that is the ' $x$ ' and ' $y$ ' co-ordinates of the joystick had been reversed. The latter mode was used as the experimental condition. No practice in the latter condition was allowed before testing began, because we were interested in measuring the subjects' baseline performance levels. The data were collected under an experimental condition that allowed one to measure the capacity of the subject to learn how to adapt to a sensory change (motor adaptation). In this condition, subjects had to reach targets presented at random locations in the IM condition. The subjects were required to perform 12 blocks of 64 trials each (a total of 768 attempts during a period of approximately $40 \mathrm{~min}$ ) under this experimental condition. The results of a pilot study involving 10 university students (data not shown) have demonstrated that subjects can attain an asymptotic performance level in the number of trials used in the present study protocol.

\section{Neuropsychological Tests}

To rule out a drug-induced generalized impairment such as sedation, two neuropsychological tests, a Purdue Pegboard test measuring motor dexterity and a D2 test evaluating attention capacities, were administered pre- and posttreatment with placebo or amantadine (Brickenkamp, 1981; Reddon et al, 1988).

Table I Subjects' Characteristics and Neuropsychological Findings

\begin{tabular}{|c|c|c|c|c|c|c|c|}
\hline & \multirow{3}{*}{ Age } & \multirow{3}{*}{ Sex } & \multirow{3}{*}{ Education level } & \multicolumn{4}{|c|}{ Neuropsychological tests } \\
\hline & & & & \multicolumn{2}{|c|}{ Purdue Pegboard $^{\mathrm{a}}$} & \multicolumn{2}{|c|}{$\mathrm{D2}^{\mathrm{b}}$} \\
\hline & & & & Pre- & Post- & Pre- & Post- \\
\hline Placebo group & $24.9 \pm 0.3$ & Six $F$, seven $M$ & $17.5 \pm 0.1$ & $15.5 \pm 1.6$ & $15.8 \pm 1.1$ & $93.0 \pm 11.8$ & $97.6 \pm 4.4$ \\
\hline
\end{tabular}

All values are expressed as mean \pm SEM, pre- and post- refer to drug administration.

aOnly data for the preferred hand are shown.

bercentile rank (PR) values are presented.

'No difference between amantadine and placebo-treated groups (Student's t-test). 


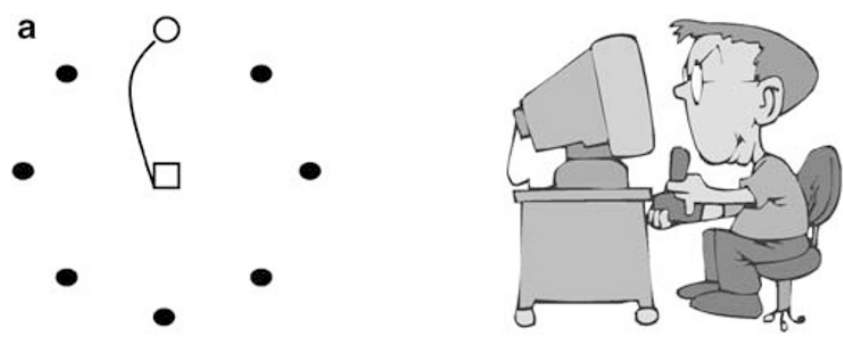

b

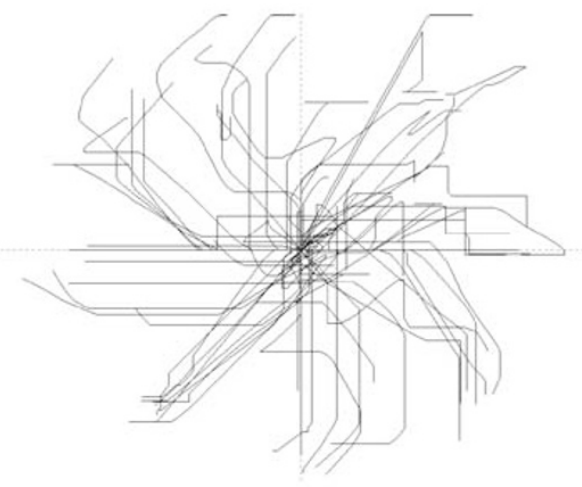

C

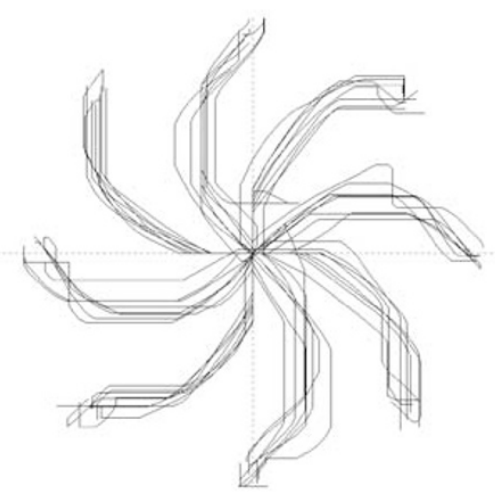

Figure I Motor-adaptation learning. (a) Figure illustrating the motoradaptation task, in which subjects are asked to reach one of the eight targets, following a curved line with a joystick in the inversed mode; that is, the ' $x$ ' and ' $y$ ' coordinates of the joystick had been reversed. (b) Early in training, movements of the curser had significant deviations from the elliptical path. (c) Late in the training, movements of the curser followed essentially a straight elliptical line.

\section{Pharmacological Manipulation}

Both amantadine (two capsules of $100 \mathrm{mg}$ each) or matching placebo were given orally in a double-blind design. In both groups, the motor-learning task was initiated $3 \mathrm{~h}$ after the drug administration to allow a maximal blood concentration for amantadine to be reached. The amantadine dose used in this experiment is equivalent to the dosage required for the prophylaxis and treatment of uncomplicated influenza A virus illness. In human subjects, maximum plasma amantadine concentrations are directly correlated for doses up to $200 \mathrm{mg} /$ day. At this dosage, amantadine is generally well tolerated, but transient side effects such as nausea, dizziness (lightheadedness), and insomnia may occur. To assay the plasma amantadine concentration, blood samples were taken $3 \mathrm{~h}$ after administration and the plasma was stored at $-70^{\circ} \mathrm{C}$ until all subjects had completed the study. Amantadine concentrations were determined using a gas-chromatographic system coupled to a massselective detector in the laboratories of Merz Pharmaceuticals (Frankfurt, Germany) (Danysz et al, 1994).

\section{Statistical Analysis}

A Performance Index (PI) of the participants was calculated for each block using a mathematical formula made up of three indices of measurement: (success rate/(distance $x$ time)), where (1) success rate refers to the number of correctly completed trials measured by the ratio of the number of targets reached over the number of possible targets per block (denominator is equal to 64: number of trials per block), (2) distance is the length $(\mathrm{cm})$ covered between the starting point and the target, and (3) time represents the time (ms) measured following the first movement of the participant until reaching the target.

A separate analysis using the success rate (accuracy) was also performed. For each subject, the level of accuracy at the end of the training period (Block 12) was compared with the baseline level (Block 1), and an improvement index (the percent of change over baseline) was calculated. A positive percent change indicates that the subject improved on the motor-learning task. For both parameters (performance and accuracy), an Analysis of Variance for repeated measures (ANOVA), followed by post hoc analysis (contrast analysis), was used to reveal the statistical significance. A Pearson correlation was employed to measure the possible relation between plasma amantadine concentrations and performance level. $P<0.05$ was regarded as significant.

\section{RESULTS}

\section{Motor-learning Performance}

Figure 2 illustrates the subjects' levels of performance in both amantadine and placebo groups. When the mean performance levels on the motor-adaptation task for all subjects in the two treatment groups were compared, motor learning improved significantly from Block 2 to Block 12, regardless of the group assignment. Although the performance level in the amantadine group never reached that of the placebo group (Figure 2), the difference was not significant. However, while all subjects in the placebo group were able to make accurate movements to reach targets and all had increased performance level with practice, only a fraction of the subjects in the amantadine group did so. Indeed, almost half of the subjects (six out of 14) in the amantadine group performed at least less than one standard deviation below that of the placebo group. Thus, we divided the amantadine group into high $(n=8)$ - and low $(n=6)$ performance subgroups. When comparing these subgroups directly, the amantadine low-performance subgroup showed performance levels significantly worser than that of the amantadine high-performance subgroup $(P<0.01$; data not shown) and the placebo group $(P<0.01$; Figure $3 a)$. To verify that this effect was not related to a selection bias, a comparable group of six subjects from the placebo group with low performance levels (placebo low-performance subgroup) was also chosen. Statistical analysis of the 


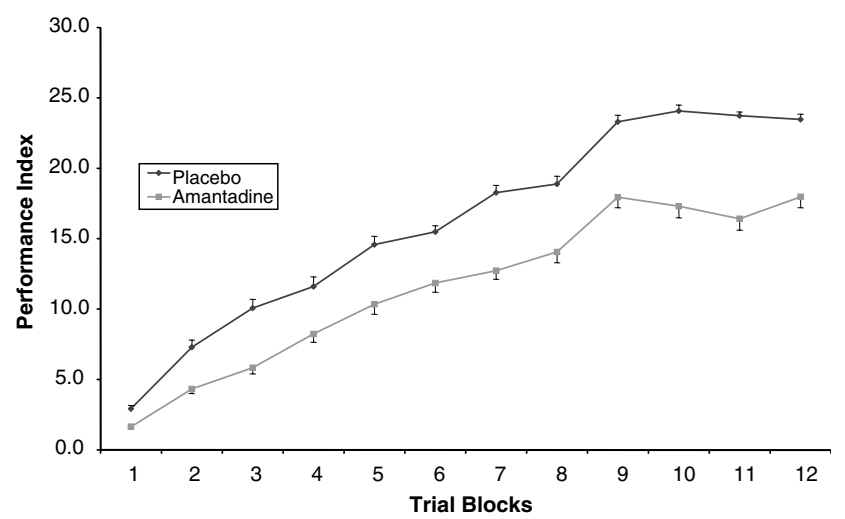

Figure 2 Motor-adaptation learning. The performance index represents the average of performance levels obtained for all participants in each treatment group during a given training block. Each block consisted of 64 consecutive trials. All values are expressed as mean \pm SEM. Comparison between amantadine and placebo groups was made using an ANOVA for repeated measures, followed by a contrast analysis (see the text for significance).
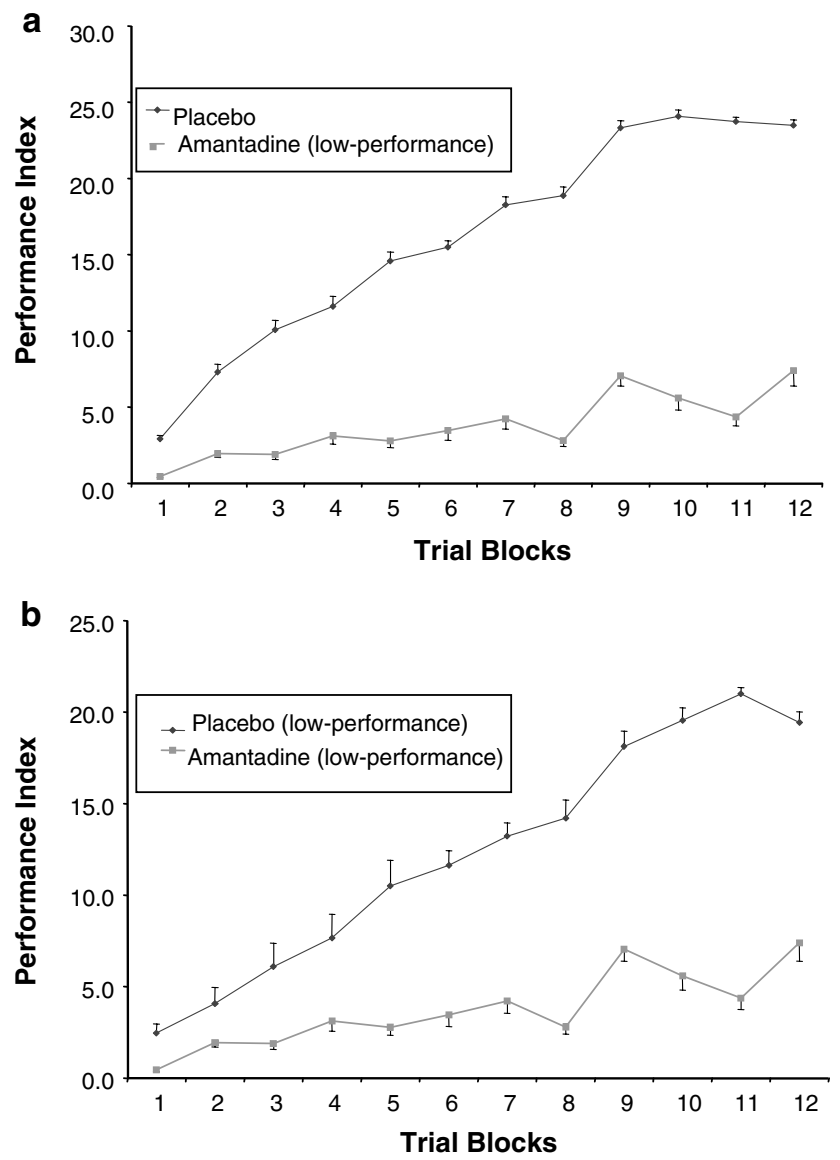

Figure 3 Motor-adaptation learning. The performance index was calculated as in Figure 2. All values are expressed as mean \pm SEM. (a) Comparison between the placebo group and the amantadine lowperformance subgroup. (b) Comparison between the amantadine lowperformance subgroup and the placebo low-performance subgroup.

comparison between the amantadine low-performance subgroup and this subgroup again showed a significant difference in motor learning $(P<0.01$; Figure $3 b)$. In contrast, no difference was found between the placebo group and the amantadine high-performance subgroup (data not shown).

\section{Motor-learning Accuracy}

Table 2 shows the subjects' levels of learning accuracy early (Block 1) and late (Block 12) in training. After 12 blocks of trials in the training period, the accuracy to perform the motor-adaptation task improved by $65.5 \pm 0.8$ and $53.46 \pm 2.1 \%$ over baseline for the placebo and amantadine groups, respectively $(P=0.11)$. However, motor-learning accuracy was lower in the amantadine low-performance subgroup than in the amantadine high-performance subgroup, the placebo group and the placebo low-performance subgroup $(P<0.01$; Table 2$)$, suggesting decreased ability of the amantadine low-performance subgroup to perform under the influence of the drug. Motor learning in the amantadine high-performance subgroup was comparable to that in the placebo group.

\section{Correlation of Motor Learning with Amantadine Concentrations and Individual Characteristics}

Peak plasma amantadine concentrations ranged from 2.09 to $4.74 \mu \mathrm{M}($ mean $=3.3 \mu \mathrm{M})$ after the acute oral administration of $200 \mathrm{mg}$ of amantadine. The amantadine lowperformance subgroup displayed mean amantadine concentrations $36 \%$ higher than the high-performance subgroup (mean $=3.8 v s 2.8 \mu \mathrm{M})(P=0.05)$, suggesting that the decreased capacity to learn the motor-adaptation task in the former subgroup could be related to higher amantadine blood levels (Figure 4). Hence, we examined whether the differential rate of learning in the amantadine group was related to their amantadine concentrations, and found a negative correlation between the performance level and the plasma concentration achieved $(r=-57, P<0.05)$ (Figure 4). Some subjects were impaired even at relatively low amantadine concentrations. Consequently, we examined whether the sensitivity to the drug was related to individual characteristics (age, gender, education level). In both amantadine and placebo groups, women appeared to perform lower than men (amantadine: $P<0.01$; placebo: $P<0.05$ ). In the amantadine group, a further analysis controlling for sex between performance index and amantadine concentrations was carried out, which revealed that the negative correlation shown in Figure 4 was no longer significant. More importantly, the subjects in the amantadine low-performance subgroup, with a gender bias $(5 \mathrm{~F}, 1 \mathrm{M})$ opposite to the amantadine high-performance subgroup $(1 \mathrm{~F}, 7 \mathrm{M})$, reached $36 \%$ higher amantadine concentrations than the latter, likely on the basis of a higher $\mathrm{mg} / \mathrm{kg}$ average dose in the former subgroup.

\section{Side Effects}

Side effects under amantadine were absent and no subject experienced drowsiness, fatigue, or dizziness. Thus, no drug-induced motor and attention impairments can account for the significant disruption of motor learning recorded in the amantadine low-performance subgroup. The Purdue Pegboard test and D2 test were administered 
Accuracy

\begin{tabular}{|c|c|c|c|c|}
\hline & & & & \\
\hline Treatment subgroups & (N: gender) & Block I & Block I2 & Improvement (\%) \\
\hline Placebo (high performance) & (seven: two F, five $M$ ) & $7.4 \pm 1.0$ & $54.3 \pm 0.7^{\mathrm{a}}$ & $73.2 \pm 1.1$ \\
\hline Amantadine (all) & (I4: six F, eight $M)$ & $4.0 \pm 0.3$ & $38.3 \pm 1.5^{\mathrm{a}}$ & $53.5 \pm 2.1$ \\
\hline Amantadine (high performance) & (eight: one $F$, seven $M$ ) & $6.3 \pm 0.5$ & $54.0 \pm 0.9^{\mathrm{a}}$ & $74.6 \pm 1.3$ \\
\hline
\end{tabular}

All values are expressed as mean \pm SEM; accuracy represents the success rate which is the number of correct trials during a given block (one block $=64$ trials). a $p<0.0$ I for comparison between pre- (block I) vs post-training (block 12) effect.

$\mathrm{b}_{p}<0.01$ for amantadine low-performance subgroup compared with amantadine high-performance subgroup, placebo group, and placebo low-performance subgroup (ANOVA for repeated measures). F: female, M: male.

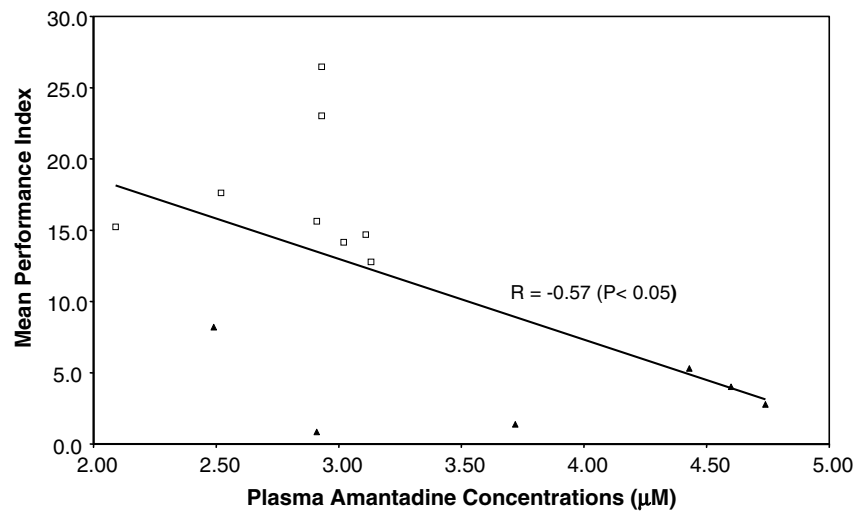

Figure 4 Relationship between motor-learning and plasma amantadine concentrations. The mean performance index, that is, the average of performance levels obtained during the 12 training blocks after a given treatment, of each individual is plotted against the corresponding plasma concentration. Additionally, the regression line of motor learning in relation to plasma level is shown. Participants with high- and low-performance levels are represented, respectively, using the symbols $(\square)$ and $(\mathbf{\Lambda})$.

pre- and post-treatment, and showed that attention and motor performance were intact under both placebo and amantadine (Table 1).

\section{DISCUSSION}

Amantadine has a plasma half-life of about $12 \mathrm{~h}$ (in young adults) and its time to peak concentration is about $3.3 \mathrm{~h}$ (Verhagen Metman et al, 2002). Therefore, its effect on motor learning should have been stable throughout this experiment. However, in our study, after a single dose of $200 \mathrm{mg}$, which is considered a strong dose, amantadine blood (and presumably brain) concentrations increased only moderately (mean: $3.3 \mu \mathrm{M}$ ). Thus, it is conceivable that the low concentration of amantadine obtained during the present study was responsible for the lack of an overall amantadine effect on motor learning, and particularly, in the high-performance subgroup. Indeed, in vitro, amantadine blocks NMDA receptors with a Ki-value at the PCPbinding site around $10 \mu \mathrm{M}$ (Kornhuber et al, 1991). Such concentrations may be reached after chronic administration in patients with Parkinson's disease, who are much older than our subjects (Kornhuber et al, 1995; Verhagen Metman et al, 1998).

Although our study sample size does not permit to draw firm conclusions about the role of gender in this type of motor learning, women appeared to perform significantly worser than men in our motor-adaptation task, in accordance with the findings of Sawaki et al (2003), who reported gender differences on a simple motor-learning paradigm using flexion-extension thumb movements. Also, it lends support to the hypothesis that female hormones may modulate neural plasticity and motor-skill learning (Maki et al, 2002; Smith et al, 1999). In our study, however, while gender may have modulated motor learning differently, it cannot explain the significant differences between amantadine low-performance and placebo low-performance subgroups, which are matched for sex distribution. Moreover, the female-predominant amantadine low-performance subgroup achieved higher mean amantadine concentrations than those in the male-predominant amantadine highperformance subgroup, likely because of our fixed, weightindependent dosing schedule, suggesting that the impairment in motor adaptation is more related to amantadine administration rather than to sex differences.

In spite of the low concentrations achieved in this experiment, amantadine still impaired motor-adaptation learning in the amantadine low-performance subgroup, as measured by our target-pointing task. This finding is in agreement with studies showing that dextrometorphan, an NMDA receptor antagonist, has a negative impact on other motor-learning paradigms, including a force-field adaptation task (Butefisch et al, 2000; Donchin et al, 2002). This effect cannot be explained by a generalized detrimental effect of such drugs on either attention (D2 test) or motordexterity performance (Purdue Pegboard), which were not affected during our study. Thus, our findings suggest that glutamatergic neurotransmission is critical for the capacity to acquire motor adaptation in humans.

It is noteworthy that higher amantadine concentrations have been achieved after chronic treatment with amantadine in Parkinson's and Huntington's disease patients (Verhagen Metman et al, 1998, 2002), and it is thus possible that such patients might have reduced ability to learn new 
motor skills. However, in one study addressing the cognitive decline in Huntington's disease, amantadine failed to alter executive functions and mental flexibility (Verhagen Metman et al, 2002). Although the current study was not designed to address the role of amantadine on motor skill learning in such neurological conditions, our data do raise the possibility that amantadine may not have the same detrimental effect on all forms of cognitive and memory functions, at least in a fraction of subjects, and that motor learning may be more sensitive to this pharmacological manipulation.

On the other hand, previous studies have reported that motor learning may not depend merely on NMDA receptor antagonism. Indeed, lorazepam (a GABA-A receptor agonist) also impairs motor learning in humans (Butefisch et al, 2000; Donchin et al, 2002). The effects of other classes of drugs, such as anticholinergics, on motor learning are not conclusive, however. Scopolamine, a muscarinic receptor antagonist, has been shown to impair use-dependent motor plasticity after repetition of thumb movements (Sawaki et al, 2002), but has failed to block motor-adaptation learning (Donchin et al, 2002). The latter finding favors the idea that the effect of amantadine is not related to its anticholinergic action exerted either directly (Nastuk et al, 1976), or indirectly through inhibition of the NMDA-evoked acetylcholine release (Stoof et al, 1992). Finally, as suggested by positron emission tomography studies (Deep et al, 1999; Moresco et al, 2002), amantadine may exert an indirect dopaminergic effect by stimulating dopa decarboxylase activity or dopamine synthesis in the brain. However, the latter proposal cannot explain our finding because amantadine at therapeutic doses does not substantially increase extracellular brain levels of dopamine or its metabolites (Stoof et al, 1992; Quack et al, 1995). Furthermore, an increase in dopamine release is likely to improve motor learning rather than dampening it, as suggested by the beneficial effect of D-amphetamine on use-dependent plasticity (Butefisch et al, 2002). Thus, the effect of amantadine on motor learning seen in this study appears largely attributable to antagonism of the glutamatergic NMDA receptors.

While the brain is capable of multiple forms of plasticity which are governed, at least in part, by independent mechanisms (Grossman et al, 2002), the acquisition of a motor skill is most likely due to LTP-like mechanisms (Hess et al, 1996). This view is supported by the fact that drugs inhibiting LTP induction also impair synaptic changes, which occur in response to motor learning such as forcefield adaptation (Donchin et al, 2002). Interestingly, lamotrigine, a drug that blocks voltage-gated $\mathrm{Na}^{+}$and $\mathrm{Ca}^{2+}$ channels (Lees and Leach, 1993) without affecting LTP induction (Xiong and Stringer, 1997), failed to reduce motor learning (Butefisch et al, 2000; Donchin et al, 2002). In our study, it is possible that amantadine interfered with changes in synaptic efficacy, explaining why it blocked motoradaptation learning. This constitutes supportive evidence for the idea that normal NMDA receptor function is important for the acquisition, not only of explicit, but also of implicit learning both in animals and humans (Martin et al, 2000; McGaugh, 2002).

Based on animal and human work, several brain structures, including the striatum, cerebellum, and motor cortical areas of the frontal lobe, are thought to be critical for the acquisition and/or retention of skilled motor behaviors (Doyon and Ungerleider, 2002; Doyon et al, 2003). Previous studies have reported possible LTP induction in the primary motor cortex (Hess et al, 1996; Butefisch et al, 2000) or the cerebellum (Lu et al, 1998; Hansel et al, 2001; Donchin et al, 2002). We cannot rule out that the cerebral plasticity associated with the motor-adaptation task used here has taken place in the striatum. Evidence supporting the role of the striatum in motor-skill learning comes from impairments found in patients with striatal dysfunction, including Parkinson's and Huntington's diseases (Willingham and Koroshetz, 1993; Doyon et al, 1997, 1998), as well as from neurophysiological studies (Graybiel, 1995; Charpier and Deniau, 1997), and lesion experiments in rodents (McDonald and White, 1993). Furthermore, the striatum is the point of entry of information into the basal ganglia, and it plays an important role in motor control and habit learning (Graybiel, 2000). The neocortex and thalamus (Parent and Hazrati, 1995) provide the major excitatory inputs to the striatal medium spiny projection neurons, where both glutamate and dopamine terminals converge (Bouyer et al, 1984; Freund et al, 1984). Finally, in the striatum, repetitive stimulation of corticostriatal fibers causes a massive release of glutamate and produces LTP (Centonze et al, 1999; Spencer and Murphy, 2000); a process similar to that seen during learning (Charpier and Deniau, 1997; Mazzucchelli et al, 2002). Thus, in our study, amantadine may have blocked striatal NMDA receptors and subsequent LTP induction required for motor learning. However, the precise site of action of amantadine on motor adaptation is not known and further studies combining pharmacological manipulations with modern brain-imaging techniques such as PET or fMRI may be interesting in this regard.

In conclusion, the present results argue for a role of central glutamatergic transmission, particularly NMDA receptors, in the acquisition of motor-adaptation learning in humans. Further studies in patients treated chronically with amantadine or multiple-dose studies in healthy volunteers are needed to confirm these predictions. Moreover, interactions with other neurotransmitters such as dopamine should be investigated in future research.

\section{ACKNOWLEDGEMENTS}

We wish to thank Bernd Eilbacher (Merz Pharmaceuticals, Frankfurt, Germany) for conducting the amantadine concentration assays, Kate Hanraty for her excellent technical assistance, and our volunteers for their participation. A Hadj Tahar holds a postdoctoral fellowship from the Foundation de l'Institut de Gériatrie de Montréal. This work was supported by the National Science and Engineering Research Council of Canada (to JD).

\section{REFERENCES}

Blanchet PJ, Metman LV, Chase TN (2003). Renaissance of amantadine in the treatment of Parkinson's disease. Adv Neurol 91: 251-257.

Bliss TV, Collingridge GL (1993). A synaptic model of memory: long-term potentiation in the hippocampus. Nature 361: 31-39. 
Bouyer JJ, Park DH, Joh TH, Pickel VM (1984). Chemical and structural analysis of the relation between cortical inputs and tyrosine hydroxylase-containing terminals in rat neostriatum. Brain Res 302: 267-275.

Brickenkamp R (1981). Test d2: Aufmerk-samkeits-Belastungs-Test (Handanweisung, 7th edn.) [Test d2: Concentration-Endurance Test: Manual, 5th edn.]. Verlag für Psychologie Dr. C.J. Hogrefe: Göttingen.

Butefisch C, Hummelsheim H, Denzler P, Mauritz KH (1995). Repetitive training of isolated movements improves the outcome of motor rehabilitation of the centrally paretic hand. J Neurol Sci 130: $59-68$.

Butefisch CM, Davis BC, Sawaki L, Waldvogel D, Classen J, Kopylev L et al (2002). Modulation of use-dependent plasticity by d-amphetamine. Ann Neurol 51: 59-68.

Butefisch CM, Davis BC, Wise SP, Sawaki L, Kopylev L, Classen J et al (2000). Mechanisms of use-dependent plasticity in the human motor cortex. Proc Natl Acad Sci USA 97: 3661-3665.

Centonze D, Calabresi P, Giacomini P, Bernardi G (1999). Neurophysiology of Parkinson's disease: from basic research to clinical correlates. Clin Neurophysiol 110: 2006-2013.

Centonze D, Saulle E, Bernardi G, Calabresi P (2001). Receptor and post-receptor mechanisms of ischemic long-term potentiation in the striatum. Funct Neurol 16(Suppl): 149-152.

Charpier S, Deniau JM (1997). In vivo activity-dependent plasticity at cortico-striatal connections: evidence for physiological long-term potentiation. Proc Natl Acad Sci USA 94: 7036-7040.

Danysz W, Gossel M, Zajaczkowski W, Dill D, Quack G (1994). Are NMDA antagonistic properties relevant for antiparkinsonianlike activity in rats? - case of amantadine and memantine. $J$ Neural Transm 7: 155-166.

Deep P, Dagher A, Sadikot A, Gjedde A, Cumming P (1999). Stimulation of dopa decarboxylase activity in striatum of healthy human brain secondary to NMDA receptor antagonism with a low dose of amantadine. Synapse 34: 313-318.

Donchin O, Sawaki L, Madupu G, Cohen LG, Shadmehr R (2002). Mechanisms influencing acquisition and recall of motor memories. J Neurophysiol 88: 2114-2123.

Doyon J, Ungerleider LG (2002). Functional anatomy of motor skill learning. In: Squire LR, Schacter DL (eds.). Neuropsychology of Memory. Guilford Press: New York. pp 225-238.

Doyon J, Gaudreau D, Laforce Jr R, Castonguay M, Bedard PJ, Bedard F et al (1997). Role of the striatum, cerebellum, and frontal lobes in the learning of a visuomotor sequence. Brain Cogn 34: 218-245.

Doyon J, Laforce Jr R, Bouchard G, Gaudreau D, Roy J, Poirier M et al (1998). Role of the striatum, cerebellum and frontal lobes in the automatization of a repeated visuomotor sequence of movements. Neuropsychologia 36: 625-641.

Doyon J, Penhune V, Ungerleider LG (2003). Distinct contribution of the cortico-striatal and cortico-cerebellar systems to motor skill learning. Neuropsychologia 41: 252-262.

Flament D, Ellermann JM, Kim SG, Ugurbil K, Ebner TJ (1996). Functional magnetic resonance imaging of cerebellar activation during the learning of a visuomotor dissociation task. Human Brain Map 4: 210-226.

Freund TF, Powell JF, Smith AD (1984). Tyrosine hydroxylaseimmunoreactive boutons in synaptic contact with identified striatonigral neurons, with particular reference to dendritic spines. Neuroscience 13: 1189-1215.

Graybiel AM (1995). Building action repertoires: memory and learning functions of the basal ganglia. Curr Opin Neurobiol 5: 733-741.

Graybiel AM (2000). The basal ganglia. Curr Biol 10: R509-R511. Grossman AW, Churchill JD, Bates KE, Kleim JA, Greenough WT (2002). A brain adaptation view of plasticity: is synaptic plasticity an overly limited concept? Prog Brain Res 138: 91-108.
Hansel C, Linden DJ, D’Angelo E (2001). Beyond parallel fiber LTD: the diversity of synaptic and non-synaptic plasticity in the cerebellum. Nat Neurosci 4: 467-475.

Hess G, Aizenman CD, Donoghue JP (1996). Conditions for the induction of long-term potentiation in layer II/III horizontal connections of the rat motor cortex. J Neurophysiol 75: $1765-1778$.

Jackson A, Lemon RN (2001). Motor control: forcing neurons to change. Curr Biol 11: R708-R709.

Kitagawa H, Nishimura Y, Yoshioka K, Lin M, Yamamoto T (1997). Long-term potentiation and depression in layer III and $\mathrm{V}$ pyramidal neurons of the cat sensorimotor cortex in vitro. Brain Res 751: 339-343.

Kornhuber J, Bormann J, Hubers M, Rusche K, Riederer P (1991). Effects of the 1-amino-adamantanes at the MK-801-binding site of the NMDA-receptor-gated ion channel: a human postmortem brain study. Eur J Pharmacol 206: 297-300.

Kornhuber J, Quack G, Danysz W, Jellinger K, Danielczyk W, Gsell W et al (1995). Therapeutic brain concentration of the NMDA receptor antagonist amantadine. Neuropharmacology 34: 713-721.

Lees G, Leach MJ (1993). Studies on the mechanism of action of the novel anticonvulsant lamotrigine (Lamictal) using primary neurological cultures from rat cortex. Brain Res 612: 190-199.

Li CS, Padoa-Schioppa C, Bizzi E (2001). Neuronal correlates of motor performance and motor learning in the primary motor cortex of monkeys adapting to an external force field. Neuron 30: 593-607.

Lu X, Hikosaka O, Miyachi S (1998). Role of monkey cerebellar nuclei in skill for sequential movement. J Neurophysiol 79: 2245-2254.

Maki PM, Rich JB, Rosenbaum RS (2002). Implicit memory varies across the menstrual cycle: estrogen effects in young women. Neuropsychologia 40: 518-529.

Malenka RC, Nicoll RA (1999). Long-term potentiation-a decade of progress? Science 285: 1870-1874.

Martin SJ, Grimwood PD, Morris RG (2000). Synaptic plasticity and memory: an evaluation of the hypothesis. Annu Rev Neurosci 23: 649-711.

Mazzucchelli C, Vantaggiato C, Ciamei A, Fasano S (2002). Knockout of ERK1 MAP kinase enhances synaptic plasticity in the striatum and facilitates striatal-mediated learning and memory. Neuron 34: 807-820.

McDonald RJ, White NM (1993). A triple dissociation of memory systems: hippocampus, amygdala, and dorsal striatum. Behav Neurosci 107: 3-22.

McGaugh JL (2002). Memory consolidation and the amygdala: a systems perspective. Trends Neurosci 25: 456-461.

Moresco RM, Volonte MA, Messa C, Gobbo C, Galli L, Carpinelli A et al (2002). New perspectives on neurochemical effects of amantadine in the brain of parkinsonian patients: a PET[(11)C]raclopride study. J Neural Transm 109: 1265-1274.

Nastuk WL, Su P, Doubilet P (1976). Anticholinergic and membrane activities of amantadine in neuromuscular transmission. Nature 264: 76-79.

Nudo RJ, Wise BM, SiFuentes F, Milliken GW (1996). Neural substrates for the effects of rehabilitative training on motor recovery after ischemic infarct. Science 272: 1791-1794.

Oldfield RC (1971). The assessment and analysis of handedness: the Edinburgh inventory. Neuropsychologia 9: 97-113.

Parent A, Hazrati LN (1995). Functional anatomy of the basal ganglia. I. The cortico-basal ganglia-thalamo-cortical loop. Brain Res Brain Res Rev 20: 91-127.

Quack G, Hesselink M, Danysz W, Spanagel R (1995). Microdialysis studies with amantadine and memantine on pharmacokinetics and effects on dopamine turnover. J Neural Transm Suppl 46: 97-105. 
Reddon JR, Gill DM, Gauk SE, Maerz MD (1988). Purdue Pegboard: test-retest estimates. Percept Motor Skills 66: 503-506.

Sawaki L, Boroojerdi B, Kaelin-Lang A, Burstein AH, Butefisch $\mathrm{CM}$, Kopylev L et al (2002). Cholinergic influences on usedependent plasticity. J Neurophysiol 87: 166-171.

Sawaki L, Yaseen Z, Kopylev L, Cohen LG (2003). Age-dependent changes in the ability to encode a novel elementary motor memory. Ann Neurol 53: 521-524.

Shadmehr R, Holcomb HH (1997). Neural correlates of motor memory consolidation. Science 277: 821-825.

Smith MJ, Kell JC, Greenberg BD, Adams LF, Schmidt PJ, Rubinow DA et al (1999). Menstrual cycle effects on cortical excitability. Neurology 53: 2069-2072.

Spencer JP, Murphy KP (2000). Bi-directional changes in synaptic plasticity induced at corticostriatal synapses in vitro. Exp Brain Res 135: 497-503.

Stoof JC, Booij J, Drukarch B, Wolters EC (1992). The antiparkinsonian drug amantadine inhibits the $N$-methyl-D-aspartic acid-evoked release of acetylcholine from rat neostriatum in a non-competitive way. Eur J Pharmacol 213: 439-443.

Verhagen Metman L, Del Dotto P, van den Munckhof P, Fang J, Mouradian MM, Chase TN (1998). Amantadine as treatment for dyskinesias and motor fluctuations in Parkinson's disease. Neurology 50: 1323-1326.

Verhagen Metman L, Morris MJ, Farmer C, Gillespie M, Mosby K, Wuu J et al (2002). Huntington's disease: a randomized, controlled trial using the NMDA-antagonist amantadine. $\mathrm{Neu}$ rology 59: 694-699.

Willingham DB (1998). A neuropsychological theory of motor skill learning. Psychol Rev 105: 558-584.

Willingham DB, Koroshetz WJ (1993). Evidence for dissociable motor skills in Huntington's disease patients. Psychobiology 21: 173-182.

Xiong ZQ, Stringer JL (1997). Effects of felbamate, gabapentin and lamotrigine on seizure parameters and excitability in the rat hippocampus. Epilepsy Res 27: 187-194. 